COMECHINGONIA. REVISTA DE ARQUEOLOGÍA Número 16, primer semestre de 2012, pp. 31-51, Córdoba

ISSN 0326-7911

\title{
INTERCAMBIO Y CARAVANAS DE LLAMAS EN EL SUR ANDINO (3000-1000 AP)
}

\author{
Hugo D. Yacobaccio
}

CONICET-Universidad de Buenos Aires. E-mail: hdyacobaccio@gmail.com

Presentado el: 31/01/2012 - Aceptado 20/04/2012

\begin{abstract}
Resumen
El propósito de este trabajo es discutir algunos aspectos relacionados con los sistemas de intercambio en el Centro Sur Andino, particularmente la Puna de Atacama, la Quebrada de Humahuaca y sectores adyacentes de las yungas para el período entre los 3000 y 1000 AP. Para ello se plantea que el intercambio solo es posible como consecuencia de una estructura que pueda garantizar su efectividad y continuidad en el plano social y que esto es llevado a cabo por acciones colectivas en situaciones estructuradas. Se revisarán casos de análisis para discutir la naturaleza del tráfico de bienes que comprenden la distribución de las obsidianas, las cerámicas San Francisco y La Isla. Estos casos serán útiles para discutir los mecanismos, agentes y organización política del intercambio. Por último, a partir de estas cuestiones teóricas y fácticas trataremos de ofrecer una serie de explicaciones plausibles acerca de las características del sistema de intercambio.
\end{abstract}

Palabras claves: Intercambio, Caravanas de llamas, Puna y Quebrada, Formativo.

\begin{abstract}
The goal of this paper is to discuss some aspects of the exchange systems in the South Central Andes, particularly the Puna de Atacama, the Quebrada de Humahuaca and adjacent areas of the Yungas for the period between 3000 and 1000 BP. Exchange is only possible out of a structure which could warrant it effectiveness and continuity in the social level, and this is accomplished by collective actions in structured situations. Three test cases will be reviewed to discuss the nature of the exchange of goods comprising the distribution of obsidian, San Francisco and La Isla ceramics. These cases will be useful to discuss the mechanisms, agents and political organization of the exchange. Finally, out of the theoretical and factual issues, we will try to offer a number of plausible explanations about the characteristics of the exchange system.
\end{abstract}

Keywords: Exchange, Llama Caravans, Puna and Quebrada, Formative.

\section{Introducción}

Las visiones tradicionales de la arqueología de los Andes Centro Sur relacionaron los comienzos de la complejidad social con la introducción de la agricultura y la cerámica. Más aún, frecuentemente fue asumido que esas innovaciones fueron la consecuencia de la difusión de ideas y de la migración de pueblos desde el altiplano boliviano, especialmente desde la cuenca del Lago Titicaca (González y Pérez 1972; Núñez Regueiro 1974). Este pe- 
ríodo, conocido como Formativo en la terminología arqueológica tradicional más aceptada, incluye algunos rasgos tales como agricultura o alguna otra economía productiva, vida sedentaria en aldeas pequeñas, sistemas extendidos de intercambio y alta variabilidad en la organización social que iría desde comunidades igualitarias hasta jerarquizadas habiéndose planteado, inclusive, para ciertas áreas la existencia de desigualdades sociales heredables (Olivera 2001; Scattolin 2007).

El término Formativo en realidad ha sido tomado muchas veces como un atajo para resumir en una sola palabra la serie de elementos presentes en las sociedades surandinas entre los 3000 y 1000 años AP (por ejemplo, Olivera y Grant 2009: 100-101). La connotación dada al mismo en este trabajo es totalmente cronológica y no resume rasgos culturales. Por lo que sabemos hoy día la tecnología cerámica fue introducida en la Puna entre los 3200 y $2900 \mathrm{AP}$ y los cambios tecnológicos asociados con la agricultura están datados en $2400 \mathrm{AP}$, junto con la aparición de las primeras aldeas circumpuneñas fuera del Salar de Atacama (allí son anteriores). El propósito de este trabajo es revisar algunos casos de interés para discutir la naturaleza del tráfico de bienes en una porción de los Andes Centro Sur para el período indicado. Para ello pasaremos revista a algunas cuestiones teóricas y fácticas tratando de ofrecer una explicación plausible acerca de las características del sistema de intercambio, tomando una muestra diversa para explorar este dominio conceptual (Smith y Peregrine 2012). Esta muestra permitirá analizar el intercambio en distintos momentos para una misma región.

Los sistemas de intercambio han sido destacados como un factor principal en la formación de las sociedades complejas (Ryden 1944; Núñez et al. 2007; Berenguer Rodríguez 2004), incluso por encima de la metalurgia (Trigger 2006). En The Oxford Companion to Archaeology se ha definido intercambio "as the transfer of people, food, raw materials, objects, rights and privileges, or ideas between two nodes, which may be individuals, social groups, or, in its loosest sense, places" (Torrence 1996:719). Esta amplia definición involucra una gran cantidad de diferentes tipos de interacciones humanas. Sin embargo, siguiendo a Polanyi (1977) el intercambio solo es posible como consecuencia de una estructura que pueda garantizar su efectividad y continuidad en el plano social. Esto no puede ser llevado a cabo por el individuo, sino por acciones colectivas en situaciones estructuradas. Esta línea, que adoptaremos en este trabajo, sigue el camino inaugurado por Marcel Mauss (1971 [1923]) al definir el "sistema del don" en el cual el intercambio se localiza en amplios contextos socioculturales, considerando que en todas las sociedades existe un vínculo entre las relaciones sociales y el intercambio (Carrier 2008). El intercambio es básicamente un movimiento de bienes entre la gente, pero esto no significa que los bienes intercambiados sean de la misma naturaleza o equivalentes. Ya el clásico planteo de Malinowski (1973[1922]) señalaba, al referirse al sistema Kula, que si bien este es de gran envergadura y está altamente ritualizado, junto con él funcionaba un "comercio normal de mercancías útiles e indispensables". Es decir, que por los mismos circuitos y en las mismas ocasiones dos clases de intercambio eran realizados simultáneamente, uno vasto y ritualizado de brazaletes y collares y otro que quizás lo podríamos denominar más mundano o utilitario (a falta de un mejor término). Esta situación de doble circulación simultánea pudo darse en el pasado andino: circulación de bienes de prestigio o ritualizados y de bienes de consumo o artefactos para tareas cotidianas. En un esfuerzo por tipificar los modos de interacción Nielsen (2006) propuso dos modalidades definidas como "tráfico especializado" y "tráfico incorporado". El primero fue definido como el traslado de bienes y personas de una región a otra mediante actividades organizadas en función de este traslado. Ello no significa que las personas que lo realizan estén especializadas en el 
tráfico, sino que el propósito de la actividad es el tráfico de bienes. El tráfico incorporado, en cambio, designa la circulación de bienes realizado en lugares comunes donde se efectuaban otras tareas temporarias como el aprovisionamiento de obsidiana o la recolección de huevos de flamencos. Esta clase de tráfico pudo haber funcionado en algunas áreas, como las Lagunas Altoandinas, donde convergían pastores de distintos lugares para aprovisionarse estacionalmente de algunos de los recursos que ofrecía la zona.

En los Andes Centro Sur las caravanas de llamas se han propuesto como el principal vehículo para el intercambio (Núñez and Dillehay 1995[1978]), excepto la costa del Pacífico, donde se plantea que los desplazamientos transversales eran realizados por "viajeros costeros" (Pimentel et al. 2011). El patrón de movilidad implicado en la circulación de las caravanas de llamas fue definido como "movilidad giratoria" que es una modalidad de circulación circular o en espiral organizada a través de asentamientos ejes conectados por rutas fijas. Esos asentamientos tuvieron tres funciones diferentes: 1) definir la movilidad de las caravanas en un amplio territorio elongado; 2) como paradas en las cuales se reciben los bienes y se organiza su redistribución a otros lugares de la región; 3) como lugares de abastecimiento de caravanas en función de permitir la continuidad del viaje. Aunque este modelo tiene algunas limitaciones (Berenguer Rodríguez 2004: 12-15), tiene un alto valor heurístico. Recientemente, el interés despertado por la investigación del tráfico entre nodos complementa el modelo giratorio centrado en los mismos (Núñez A. y Nielsen 2011). Estos estudios internodales se basan en el análisis de la red vial y los sitios, estructuras y rasgos vinculados directamente al tráfico (Nielsen 2011:84).

La organización política que posibilita el tráfico también ha sido discutida, enfocada principalmente en la identificación de los agentes que promueven las caravanas de llamas. Se han propuesto tres opciones (Núñez 1976; Berenguer Rodríguez 2004; Aschero 2006; Nielsen 2006):

1. pastores individuales que hacen viajes como parte de sus actividades de subsistencia;

2. individuos especializados dentro de las comunidades pastoriles;

3. grupos corporativos dependientes de líderes políticos.

Durante el Formativo no hay evidencias que indiquen alguna especialización en los bienes que circularon, si no más bien lo contrario, ya que el registro arqueológico altoandino contiene una alta variabilidad de bienes originados en otras regiones (Tabla 1). Por otra parte, si bien el tráfico durante este período no parece haber tenido la intensidad de momentos posteriores, fue muy regular en el tiempo como lo muestra la circulación de las obsidianas. Si asumimos que el tráfico de bienes, tal como planteamos más arriba, se origina en acciones colectivas como parte de relaciones sociales específicas, entonces la opción 2 parece la explicación más viable para lo que se ha definido como tráfico especializado, dado que el pastoreo de camélidos es una precondición para formalizar redes de caravanas de llamas. La primera opción, por otra parte, es característica del tráfico incorporado. Pensamos, sin embargo, que esta modalidad por sí misma no puede explicar la regularidad de los intercambios en el largo plazo (en el caso de la obsidiana, unos 2400 años) y la variabilidad de los bienes intercambiados a través del tiempo, ya que los intercambios relacionados con la subsistencia están sujetos a variables contingentes tales como las fluctuaciones ambientales que afectan la producción pastoril en pequeña escala (Lupo et al. 2007). Analizaremos la viabilidad de estas opciones a lo largo del trabajo. La opción 3 ha sido discutida para momentos posteriores a los AD 1300, cuando se aprecia una mayor intensidad del tráfico de 
caravanas originadas en las tierras altas y no la discutiremos aquí (Berenguer Rodríguez 2004; Nielsen 2011; Valenzuela et al. 2011).

\begin{tabular}{|c|c|c|c|c|}
\hline Desde & Puna & $\begin{array}{c}\text { Valles } \\
\text { Mesotérmicos }\end{array}$ & Yungas & $\begin{array}{c}\text { Costa del Océano } \\
\text { Pacífico }\end{array}$ \\
\hline Puna & & $\begin{array}{l}\text { Obsidiana } \\
\text { Metales } \\
\text { Cuentas de } \\
\text { piedra }\end{array}$ & $\begin{array}{l}\text { Obsidiana } \\
\text { Metales } \\
\text { Cuentas de } \\
\text { piedra } \\
\text { Pigmento } \\
\text { rojo? }\end{array}$ & $\begin{array}{l}\text { Pieles y fibras } \\
\text { de camélidos }\end{array}$ \\
\hline $\begin{array}{c}\text { Valles } \\
\text { Mesotérmicos }\end{array}$ & $\begin{array}{l}\text { Cerámica } \\
\text { Maíz } \\
\text { Frutas } \\
\text { Zapallo } \\
\text { Ají } \\
\text { Calabaza } \\
\end{array}$ & & $\begin{array}{l}\text { Cerámica } \\
\text { Pigmento } \\
\text { rojo? }\end{array}$ & $\begin{array}{l}\text { Maíz } \\
\text { Cultivos } \\
\text { Cerámica }\end{array}$ \\
\hline Yungas & $\begin{array}{l}\text { Caracoles } \\
\text { Cerámica } \\
\text { Plumas de Aves } \\
\text { Tropicales } \\
\text { Cultivos ¿? }\end{array}$ & $\begin{array}{l}\text { Cuentas de } \\
\text { caracol } \\
\text { Plantas }\end{array}$ & & \\
\hline $\begin{array}{c}\text { Costa del Océano } \\
\text { Pacífico }\end{array}$ & $\begin{array}{l}\text { Cuentas de } \\
\text { valva }\end{array}$ & $\begin{array}{l}\text { Peces } \\
\text { Mariscos }\end{array}$ & & \\
\hline
\end{tabular}

Tabla 1. Bienes más comunes intercambiados indicando su ecorregión de origen.

El mecanismo de intercambio pudo ser del tipo down-the-line para algunos objetos durables y de gran valor como sugirió Nielsen (2004; 2006:47). Dentro de esta situación los bienes viajan por diferentes territorios a través de sucesivos intercambios sin la necesidad de que las comunidades que están en los extremos de la red de intercambio tengan contacto directo. La función redistributiva de los asentamientos eje, dentro del modelo de movilidad giratoria, es afín a este mecanismo de intercambio.

Los estudios etnográficos sobre caravanas de llamas contribuyen de manera importante para evaluar la cultura material involucrada en la circulación de bienes y para elaborar hipótesis sobre la logística de los movimientos. Pero debemos tener en cuenta que el tráfico actual es una actividad familiar de pequeña escala que llegan a incluir entre 60 y 80 llamas (Cipolletti 1984) (Figura 1). Por ello la caracterización de las caravanas del pasado no debe seguirse de manera directa. Las caravanas actuales funcionan en la estación seca y pueden hacer entre 1 y 4 viajes en el año. En condiciones normales recorren entre 15 y $25 \mathrm{~km}$ diarios, aunque excepcionalmente pueden transitar entre 35 y $40 \mathrm{~km}$. Las rutas son regulares y fijas y en los puntos terminales de cada día puede haber corrales, refugios o habitaciones para el descanso nocturno de llamas y personas (Nielsen 1997; Paz Flores 2000). Esos puntos locales son sitios de descanso nocturno también conocidos como "jaranas" o "paskanas". Por ejemplo, entre los $115 \mathrm{~km}$ entre Cerrillos (Sud Lipez) y Tupiza en Bolivia hay una jarana cada $8 \mathrm{~km}$ aproximadamente (Nielsen 1997:342). Los sitios de ocupación prolongada (uno o dos días de ocupación cada 3 ó 4 días de viaje) forman un entramado en el paisaje que 
sostiene la logística y las acciones sociales y rituales de los caravaneros fuera de sus lugares de origen. En el punto terminal de la ruta ellos intercambian sus productos (carne, charqui, fibras, cueros, sogas, bolsas, textiles o llamas vivas) por otros bienes, tales como maíz, fruta, azúcar, hojas de coca, etc.

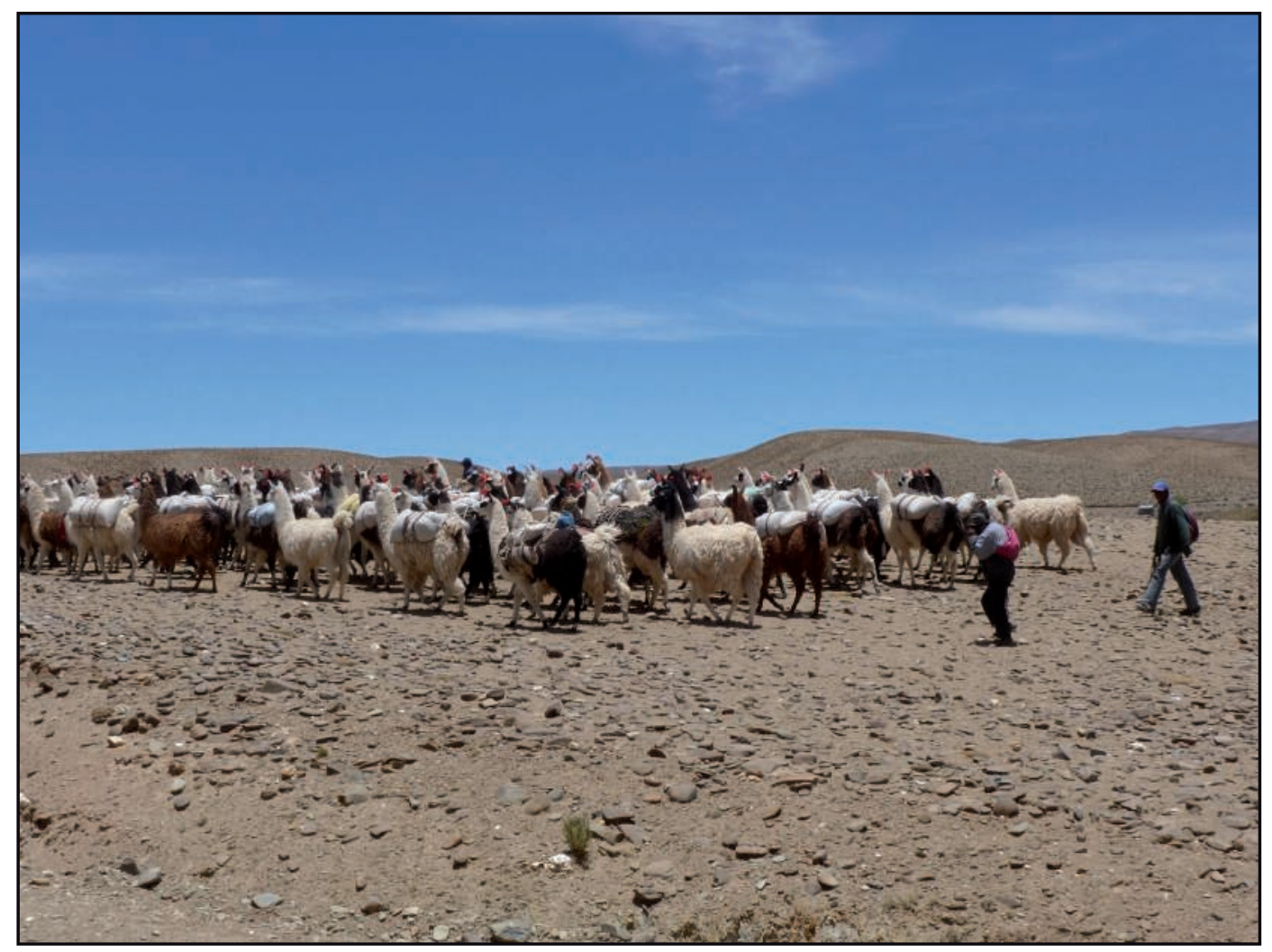

Figura 1. Caravana de llamas arribando al Noroeste Argentino desde Bolivia, 2011. Formada por alrededor de 55 llamas. Foto: Bibiana Vilá.

\section{Economía y Complejidad Social}

Tenemos evidencia de que al menos para la Puna alguna forma de complejidad social se originó dentro de las poblaciones de cazadores recolectores por lo menos desde los 4200 años AP. Esta fue el resultado de nuevas relaciones sociales surgidas de la reducción de la movilidad (inducida por factores ambientales actuantes desde los comienzos del Holoceno Medio), la especialización económica en la explotación de los camélidos y la existencia de líderes visibles que ejercían fuerte influencia y, que quizás, actuaran de catalizadores sobre la circulación de bienes exóticos (Yacobaccio 2004, 2006; Núñez et al. 2007). El proceso de domesticación local de los camélidos, al menos en la zona de Atacama (Cartajena et al. 2007; Yacobaccio 2004) se expandió gradualmente y provocó la radiación de las llamas (Lama glama) sobre toda la región y la consecuente generación de la economía de pastoreo extendida en la Puna al menos desde los 3000 años AP, como producto de la intensificación económica sobre los animales domesticados. Es bueno enfatizar que la primera variedad de llama registrada arqueológicamente es similar en tamaño a la actual llama carguera. En ese mismo momento son introducidos a la región desde zonas más bajas los primeros cultivos, 
tales como quinoa (Chenopodium quinoa), calabaza (Lagenaria siceraria), zapallo (Cucurbita $\mathrm{sp})$, oca (Oxalis sp), y maíz (Zea mays). Si bien no podemos estar seguros que algunos de ellos, como la quinoa o la oca, fueran cultivados en la Puna u obtenidos por intercambio, se registró cierto grado de procesamiento local de estos vegetales a partir del análisis de los instrumentos de molienda (Babot 2004).

Estos cambios alentaron la aparición de aldeas complejas en la Puna de Atacama. Tulán 54 es un ejemplo de este desarrollo, en parte resultado de un temprano proceso de sedentarización que caracterizó las ocupaciones de la margen sudeste del Salar de Atacama desde 4800 AP. Según Núñez et al. (2005:301) el carácter complejo del sitio se debe al desarrollo de la economía de pastoreo y a la intensificación del intercambio regional de larga distancia. La aldea tiene $2800 \mathrm{~m}^{2}$ compuestos por estructuras residenciales circulares y un pequeño templo oval de alrededor de $85-90 \mathrm{~m}^{2}$ con piso semi subterráneo. En este lugar se hallaron 24 inhumaciones de niños (entre 3 y 6 meses de edad) enterrados en 12 pozos excavados en la fundación de la estructura. Algunos de ellos tenían como acompañamiento estatuillas de oro y otros artefactos de materia prima exótica a la región. La aldea está fechada a partir de los 3030 años AP, pero aparentemente el templete se construyó posteriormente desde los 2840 AP (Núñez et al. 2005).

En el sitio hay evidencias de manufactura de cuentas de diversas materias primas, tales como mineral de cobre, valvas del Océano Pacífico (Pecten, Semele), caracoles de las yungas (Strophocheilus) y piedras semipreciosas (malaquita, turquesa, crisocola y laspislázuli). Los excavadores del sitio señalaron que las cuentas eran artefactos de prestigio utilizados como ofrenda en los enterratorios y como bienes de intercambio.

Otras aldeas en el sector oriental de la Puna, aunque menos complejas, tienen características similares. Hay presencia de estructuras circulares de piedra ubicadas en las terrazas de los ríos como Piscuno y Torre (Fernández Distel 1998) y algunas, como Matancillas, tienen construcciones para la agricultura y restos botánicos de maíz y quinoa (Muscio 2007). Todas ellas tienen cerámica exótica y evidencia de producción de cuentas; en Torre, se han detectado evidencias de actividades de metalurgia.

Dado que el pastoreo de camélidos impone cierto grado de movilidad, los pastores tempranos también utilizaron cuevas y abrigos rocosos como sitios temporarios, tales como Tomayoc, Cueva Quispe, Huirunpure (también con elementos exóticos y metalurgia) y, en los sistemas agropastoriles periféricos a la Quebrada de Humahuaca, emplearon sitios de habitación estacionales como Inca Cueva al. 1, Inca Cueva 5 y otros de alto Zapagua (Garcia 2003) o cueva de Cristóbal en Mina Aguilar.

Ahora pasaremos a presentar tres casos de circulación de bienes y a discutir las posibilidades del sistema de intercambio.

\section{Obsidiana}

Las redes de distribución de la obsidiana son buenos marcadores de circulación interregional de artefactos y materias primas y son útiles para evaluar las distancias involucradas en el tráfico, ya que sus lugares de origen pueden establecerse con alto grado de certidumbre. Investigaciones recientes (Escola 2004; Yacobaccio et al. 2002, 2004; Selenfreund et al. 2010) documentaron la existencia de más de 10 fuentes diferentes de obsidiana en los Andes Centro Sur, particularmente en el Noroeste Argentino, siendo dos de ellas regionalmente 
las más importantes debido a la gran distancia geográfica que alcanzaron y a la intensidad de su explotación.

La distribución desde estas dos importantes fuentes fue muy extensa, pero ambas tuvieron áreas de repartición discretas y mutuamente exclusivas, teniendo un alcance entre 300 y $450 \mathrm{~km}$ a partir de la fuente. Estas dos áreas de distribución se ubican en el extremo norte de la Puna (fuente Zapaleri/Laguna Blanca) (Figura 2) y la otra en el sector sur, localizada en la provincia de Catamarca (Ona/Las Cuevas). Estas áreas tuvieron una gran estabilidad temporal a partir de los 2200 años AP, aunque su empleo comenzó mucho tiempo antes. En el caso de Zapaleri/Laguna Blanca, recientemente se constató que su explotación comenzó hace unos 9700 años AP, a partir de evidencias del sitio Hornillos 2 (Susques, Jujuy).

Queremos resaltar la notable estabilidad de este patrón de circulación, que sólo cambió en tiempos incaicos, a pesar de que los bienes de obsidiana encontrados en los sitios arqueológicos varían en frecuencia. La explicación de esta persistencia tiene varios aspectos; en primer lugar, el tráfico pudo incluir no sólo materias primas, sino también artefactos

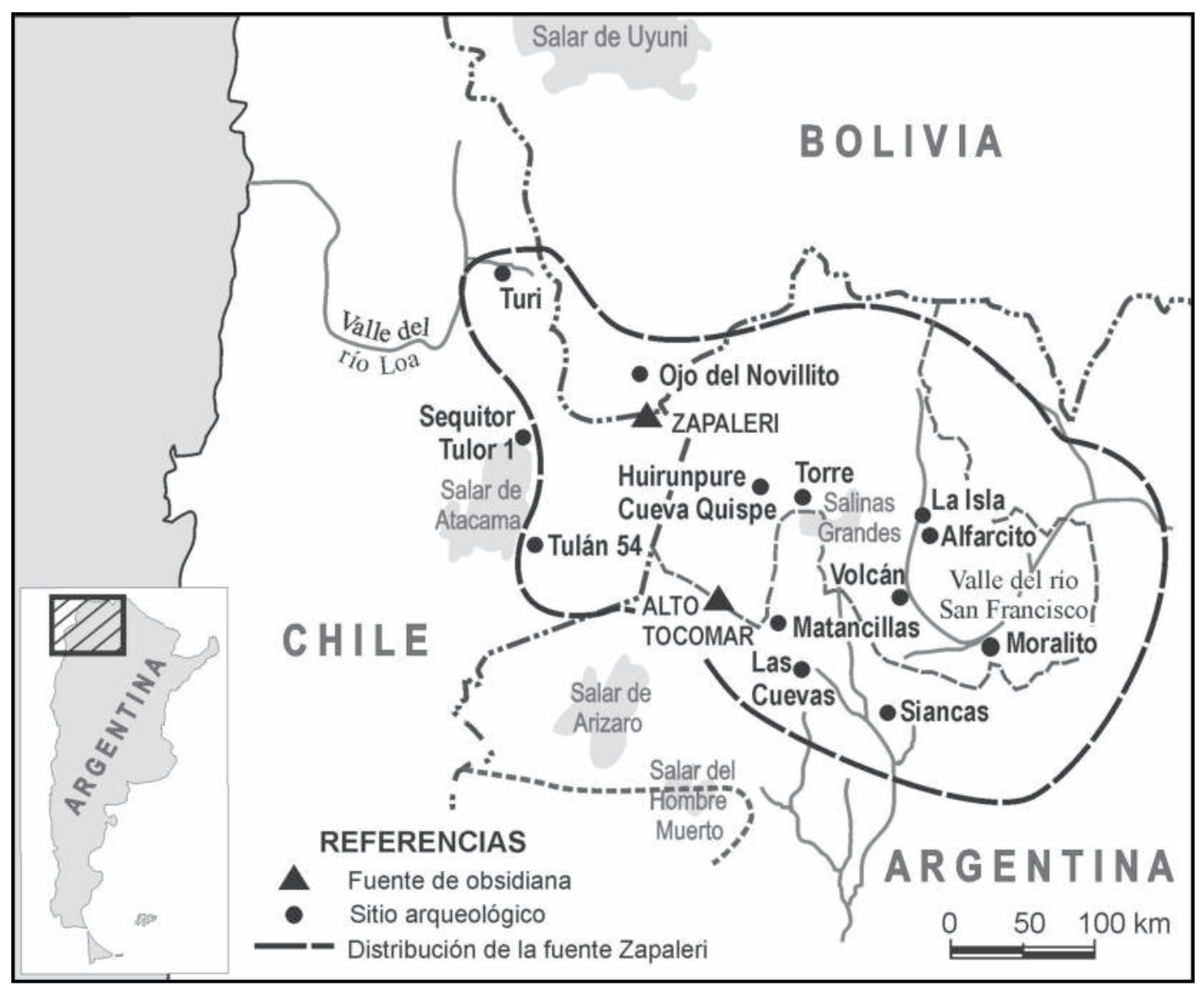

Figura 2. Mapa con el área de distribución de la obsidiana de Zapaleri/Laguna Blanca. Se indica la fuente de Tocomar y se ubican los sitios analizados en este trabajo. Sitios con cerámica SF: Moralito, Siancas, Las Cuevas, Matancillas, Volcán, Torre, Tulán 54, Ojo del Novillito, Turi, Sequitor y

Tulor 1. 
manufacturados. En segundo lugar, esto pudo ser facilitado por la introducción del arco y la flecha; en efecto, las puntas de flecha son más pequeñas y estandarizadas, por eso la obsidiana pasó a ser la materia prima predominante para su confección debido a su alta calidad para la talla. Esta situación debió ser aprovechada rápidamente por los encargados del tráfico y estos artefactos, junto a sus materias primas, fueron puestos en circulación en niveles no vistos anteriormente con los grupos de cazadores recolectores que utilizaban en mayor medida otras materias primas. Con esto queremos decir que la regularidad temporal y espacial y el predominio de la obsidiana en la tecnología de puntas de flecha del período en diferentes regiones, impide pensar que los agentes del tráfico fueran exclusivamente pastores que incidentalmente estuvieran intercambiando cosas para propósitos de subsistencia (opción 1), sino que probablemente también fueran individuos (pastores) especializados en el tráfico (opción 2), aunque una opción no contradice a la otra.

\section{Dos casos de estudio con la cerámica}

Tradicionalmente la cerámica fue el principal objetivo de los arqueólogos cuando buscaban monitorear el intercambio regional. Los estilos cerámicos regionales fueron útiles para establecer los extremos del rango geográfico en los cuales esos artefactos viajaron. Aunque el intercambio fue generalmente asumido para explicar la presencia de un estilo cerámico fuera de su supuesto centro de origen, se han propuesto modelos más complejos para explicar la expansión de algunos de ellos, por ejemplo de la cerámica San Francisco (SF) originada en el área subandina del Noroeste Argentino.

El centro de origen del complejo cultural San Francisco está aparentemente ubicado en las márgenes del río homónimo y sus tributarios en la transición entre las yungas y el bosque xerofítico del área chaqueña. La mayoría de los 37 sitios documentados para definir a este complejo cultural está localizada en la planicie aluvial del río San Francisco a 600-700 $\mathrm{m}$ de altura sobre el nivel del mar. Fechados radiocarbónicos referidos al mismo tienen un rango de $2500 \mathrm{AP}$ hasta $1400 \mathrm{AP}$, aunque esta tradición aparentemente persistió por algunas centurias más.

Los sitios del complejo San Francisco pueden ser agrupados en bases residenciales usados por largo tiempo, algunas de ellas asociadas a cementerios; sitios estacionales; sitios de actividades específicas ocupados en el corto plazo para obtener recursos limitados y montículos de función desconocida (Ortiz 2003). Se ha planteado que la economía de esta sociedad estuvo basada en la agricultura de maíz, quinoa, Amaranthaceae y porotos. También, en un sitio (Moralito) se encontró polen de coca (Erithroxylon) (Echenique y Kulemeyer 2003). Muchos sitios proveen evidencia de caza y recolección habiéndose enfatizado esta cuestión para replantear la base económica en la que se fundamentaba este complejo (Ortiz 2003). Este planteo se potenció debido a la baja frecuencia de caries y la ausencia de líneas de hipoplasia del esmalte en restos humanos provenientes del sur del río San Francisco (Seldes y Ortiz 2007). En Moralito, particularmente, se registró un variado número de especies, tales como camélidos (Lama sp), venado (Mazama sp), armadillos (Dasypus $\mathrm{sp}$, Euphractus sp), roedores (Ctenomys sp; Akodon sp), aves (Penelope sp), ranas e iguanas (Tupinambis sp). Sin embargo, no poseemos datos sobre la abundancia de estas especies en el registro arqueológico, aunque la alta riqueza específica permite suponer el empleo de una estrategia de caza generalizada. 
La distribución de cerámica SF es geográficamente amplia, extendiéndose hacia los valles mesotérmicos (Siancas), la quebrada de Humahuaca, la Puna, los oasis de San Pedro de Atacama, el río Loa y el altiplano sur de Bolivia (Ortiz 2007; Nielsen 2006) (Figura 3). La cronología de estos contextos varía entre los 2040 y 1350 AP.

Algunos autores sostienen que la expansión de esta cerámica desde su área de origen estuvo asociada a una difusión démica desde los 2000 AP aproximadamente, producida por la expansión de la frontera agrícola (Muscio 2007:124). También se ha sugerido que la ocupación más temprana, en un componente datado en 1940 AP, del sitio Volcán en la Quebrada de Humahuaca inferior, constituye una expansión de SF hacia la Quebrada (Cremonte y Fumagalli 2001).

La evidencia para afirmar la difusión démica es diferente en ambos casos. En el primero de ellos, se infirió que la tecnología agrícola puneña es causada por la expansión desde tierras bajas en base a una supuesta datación anterior de la misma en las yungas y que en su radiación incluyó la cerámica SF. En el caso de Volcán se basa exclusivamente en la presencia de cerámica en el componente más temprano del sitio. Las evidencias cerámicas merecen un análisis más detallado.

En el valle del río San Francisco han sido definidas 14 modalidades decorativas que combinan diferentes técnicas en relación con cinco tipos de acabado de la superficie (Ortiz 2003:36) (Tabla 2; Figura 3). Nos parece interesante analizar qué cantidad de esas variantes decorativas se hallaron fuera del área como una medida de la intensidad de circulación, más que tomar números absolutos o relativos de la cantidad de tiestos.

Cinco de esas modalidades decorativas fueron registrados en Volcán en un contexto que involucra, además, un gran número de fragmentos, y vasijas de gran tamaño (Cremonte y Fumagalli 2001). En todos los otros sitios (Figura 1), fueron hallados solo de 1 a 3 modalidades decorativas todas en vasijas de pequeño tamaño, excepto en Las Cuevas donde se registró la presencia de 4 modalidades (Raffino 1977). En estos sitios la mejor explicación de la presencia de cerámica SF sería por el funcionamiento de algún mecanismo de intercambio. Esta hipótesis también aplica a Matancillas en el cual se encontró una baja cantidad de modalidades decorativas; la misma cantidad que en Tulán 54. Una excepción a esta explicación queda hecha para Volcán, donde fueron halladas grandes cantidades de cerámica con homogeneidad estilística y mayor diversidad de tipos decorativos, pudiendo implicar algún tipo de desplazamiento poblacional.

\begin{tabular}{|l|}
\hline \multicolumn{1}{|c|}{ Modalidades decorativas } \\
\hline 1. Impresión unguicular \\
\hline 2. Impresión dactilar \\
\hline 3. Impresión de cuerdas vegetales \\
\hline 4. Modelado de tiras aplicadas al pastillaje \\
\hline 5. Incisión \\
\hline 6. Incisión en línea fina y gruesa \\
\hline 7. Incisión y pintura \\
\hline 8. Pintura bicolor \\
\hline 9. Pintura monocroma \\
\hline 10. Corrugado \\
\hline 11. Impresiones dactilares \\
\hline 12. Acanalado dactilar o inciso \\
\hline 13. Tiras de arcilla al pastillaje \\
\hline 14. Pintura y engobe monocromo \\
\hline
\end{tabular}

Tabla 2. Modalidades decorativas de acabado de la superficie en cerámica San Francisco (tomado con modificaciones de Ortiz 2003: Tabla 1). 

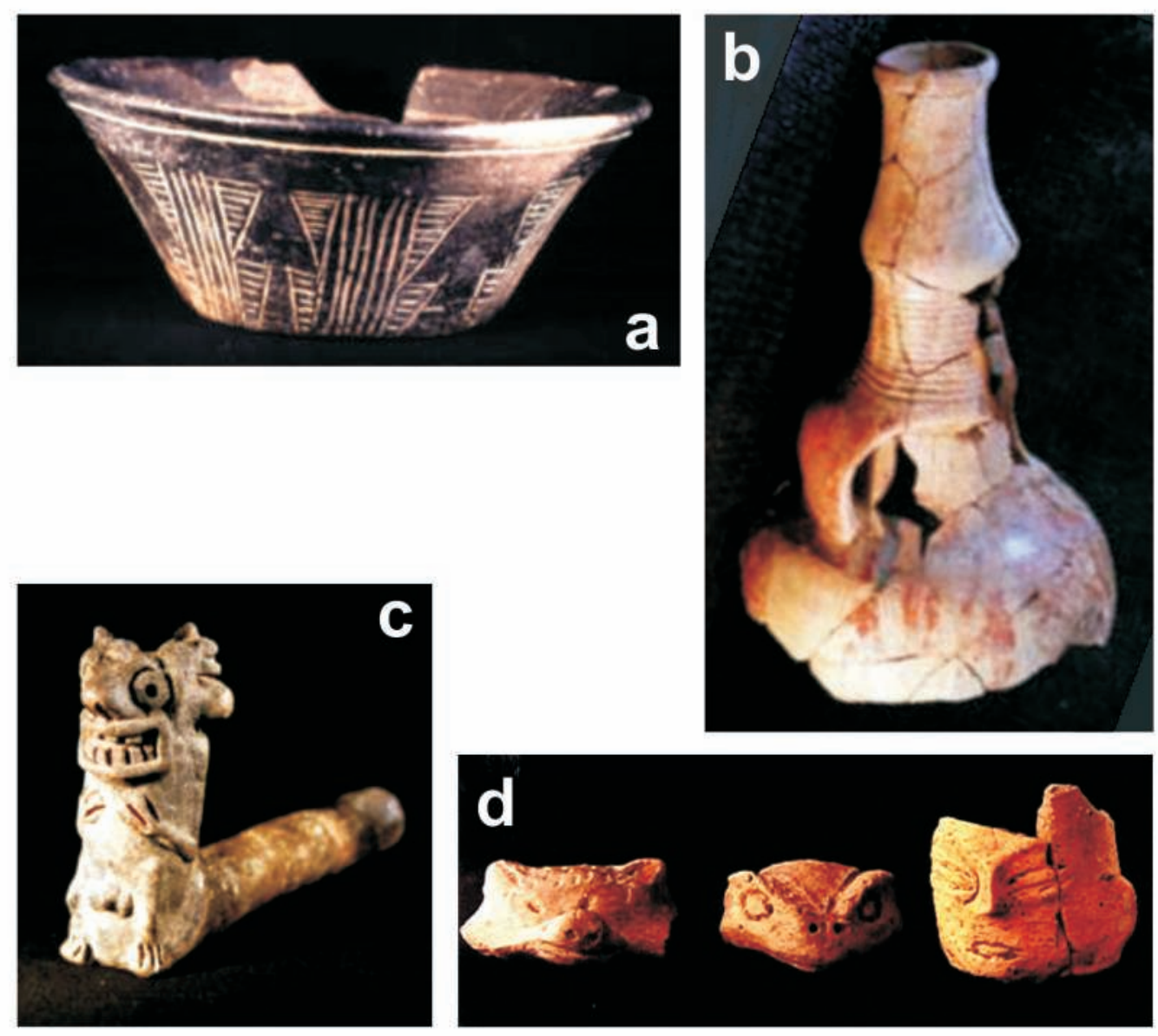

Figura 3. Cerámica San Francisco donde se aprecian algunas modalidades decorativas (inciso, inciso y pintura, modelado). Las proveniencias son (a) de Torre; (b), (c) y (d) de Moralito.

La hipótesis de intercambio en este trabajo es sostenida por la variación en la frecuencia de modalidades decorativas distribuidas en la región dado que la misma encaja con una curva de distribución fall-off (Figura 4). Esta curva muestra que los materiales intercambiados declina a medida que la distancia desde el origen aumenta. Este tipo de curva puede ser resultado de la actuación de un mecanismo de intercambio down-the-line con centros menores de redistribución, cuestión también sostenida por el modelo de movilidad giratoria. Las Cuevas, un sitio localizado cerca del área subandina, pudo haber actuado como uno de esos centros secundarios. Más aún, podemos hipotetizar que las cerámicas SF de Matancillas y Tulán 54 llegaron desde este lugar.

Pero, ¿qué pasa en el valle de San Francisco? El registro indica que sólo un pequeño grupo de puntas de proyectil de obsidiana y anillos y brazaletes de cobre fue recuperado en los sitios de este valle. Estos pocos elementos exóticos contradice la expectativa de un tráfico intenso como se esperaría de la amplia distribución de la cerámica SF fuera de la región, pero esto puede explicarse como una consecuencia del mecanismo de intercambio empleado, ya que la misma pudo haber tenido este amplio rango de dispersión a partir de 


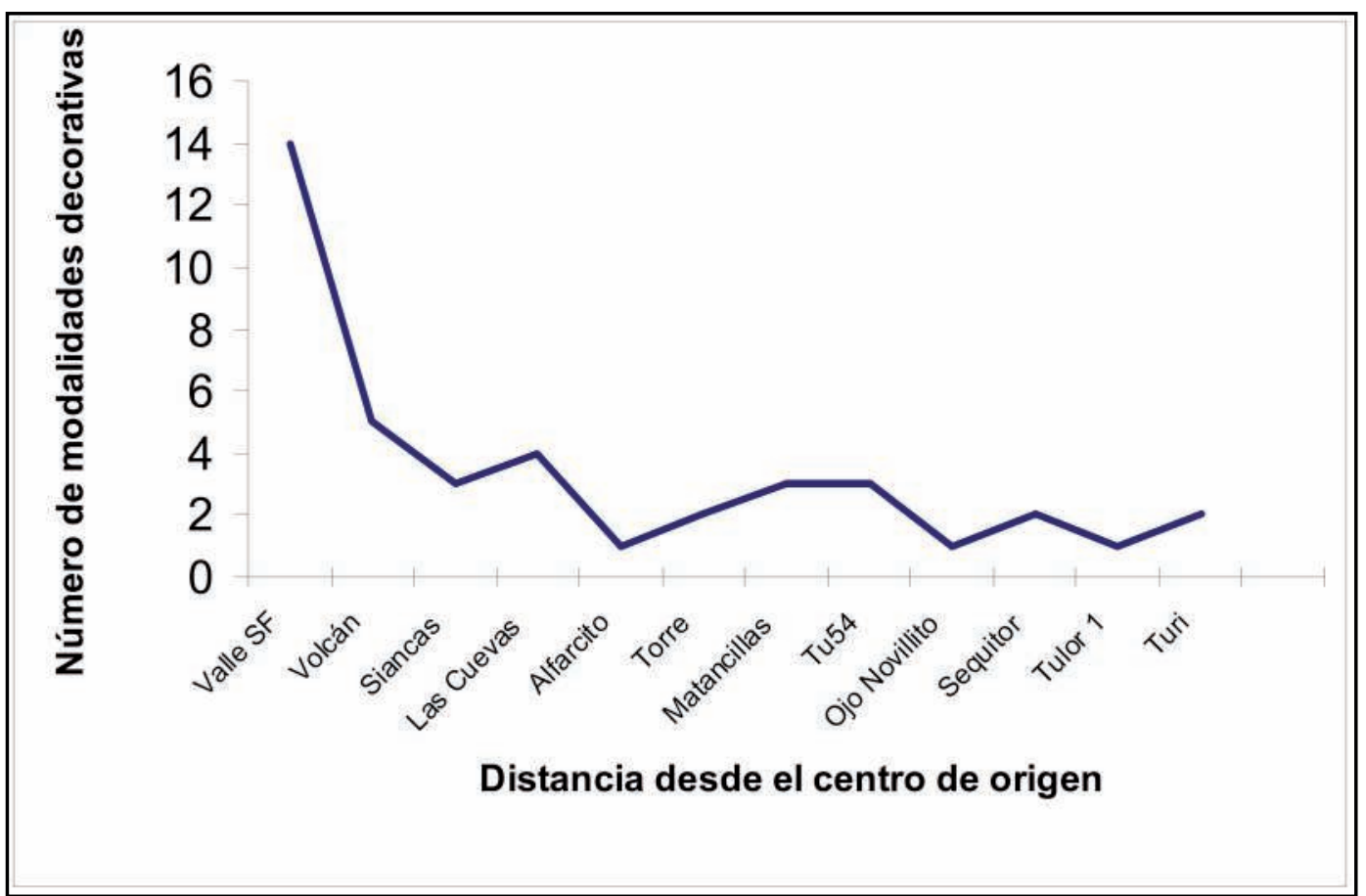

Figura 4. Curva fall-off de la distribución de modalidades decorativas de la cerámica San Francisco.

su redistribución desde centros secundarios evidenciado en la baja variabilidad de las modalidades decorativas halladas fuera de su región de origen. Tampoco puede descartarse que objetos perecibles de madera y otros materiales perecibles pudieran haber formado parte del tráfico pero, debido a las malas condiciones para su preservación, estén ausentes en el registro arqueológico del área. También los elementos importados pudieron haber sido recursos para la subsistencia, como charqui o pieles de camélido. Algunos autores piensan que el intercambio no estuvo focalizado en productos para la subsistencia sino sólo en objetos de prestigio (Ortiz 2007:320). Aunque esto no parece razonable carecemos de suficiente evidencia para abrir un juicio definitivo sobre este aspecto.

Otro caso de intercambio irregular concierne al complejo cerámico La Isla. Desde la definición de este estilo cerámico sesenta años atrás, los arqueólogos plantearon que esta particular cerámica polícroma, originada en el sector medio de la Quebrada de Humahuaca, era más temprana que las cerámicas negro sobre rojo asociadas a la ocupación de los conglomerados conocidos como Pucarás (Figura 5). Esta posición temprana puede ser sostenida por el análisis biológico de algunos individuos de La Isla, quienes presentan rasgos diferenciales respecto de las poblaciones más tardías de la Quebrada, particularmente en el tipo de deformación craneana (Cocilovo et al. 2001). Por otra parte, el descubrimiento de dos pequeñas vasijas de La Isla en tumbas de San Pedro de Atacama llevó a la conclusión de que estos objetos "...pudieron haber sido obtenidos por comercio" (Tarragó 1977:62). Estas dos piezas estaban asociadas con cerámica San Pedro negro pulido y artefactos de madera Tiwanaku que permitió estimar la edad de los contextos en AD 800. Nuevamente, como en el caso de San Francisco, no se encontró cerámica San Pedro negro pulido en el sitio epónimo de La Isla. 


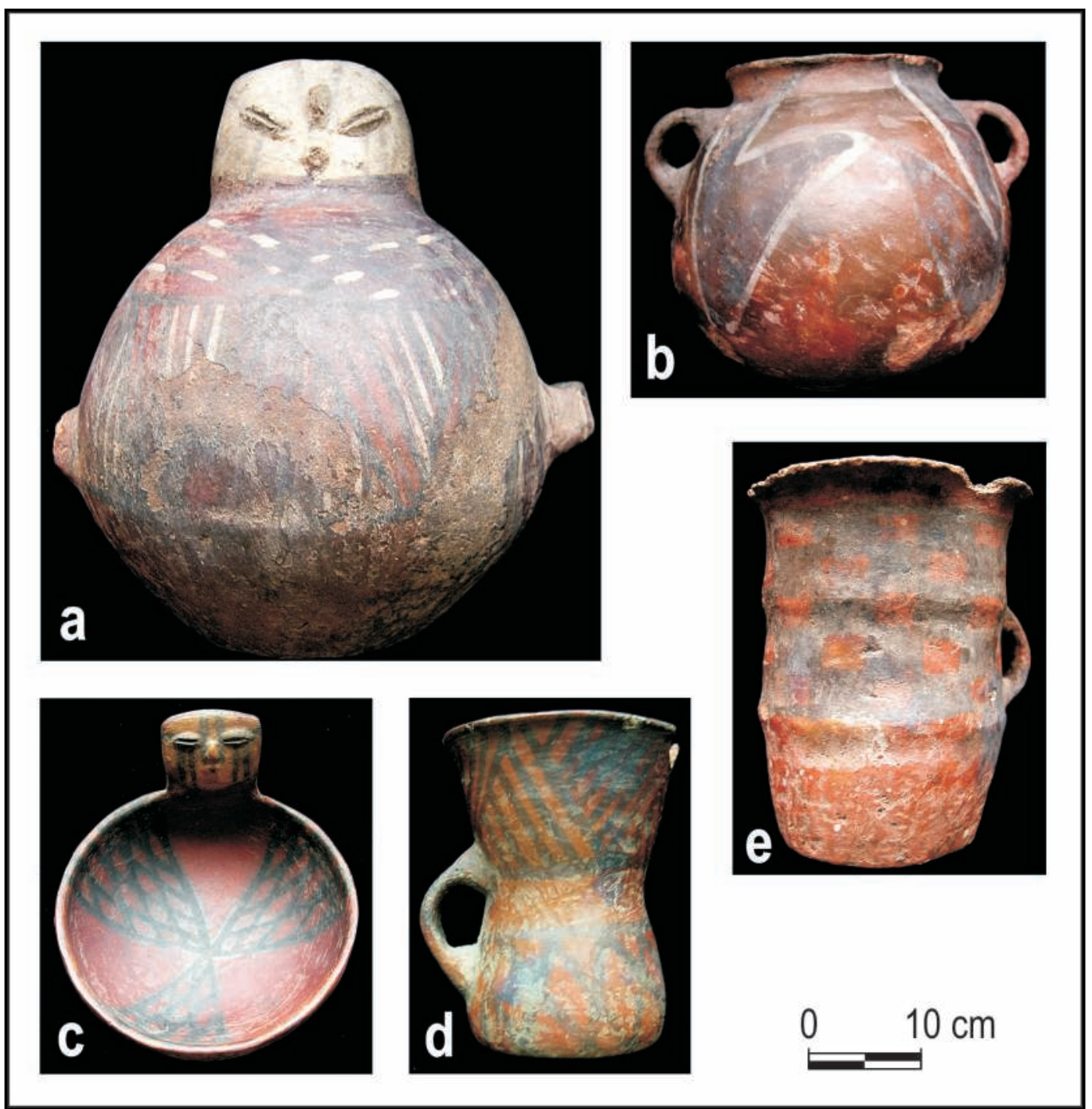

Figura 5. Cerámica La Isla. Todas del sitio epónimo, excepto (b) que es de Huacalera. El vaso (d) es muy similar al registrado por Tarragó (1977) en San Pedro de Atacama. Colección del Museo Arqueológico "Eduardo Casanova", Instituto Interdisciplinario Tilcara. Fotos del autor.

Debenedetti (1910) recuperó cientos de artefactos de 32 tumbas de este sitio. Dividió a la localidad en tres sectores, El Morro, Necrópolis A y Necrópolis B, obteniendo una gran cantidad de cerámica en complejas asociaciones $y$, siendo esta clase de artefactos, la principal ofrenda en conjunto. Aunque, ninguna de las tumbas fue datada radiocarbónicamente, las asociaciones cerámicas sugieren un gran período de uso del cementerio, parte del mismo más allá del período que estamos considerando. Tarragó et al. (2010) estiman, en base al análisis de la cerámica presente en le tumba 11 de El Morro, que dicho componente con vasos La Isla y Yavi temprano, junto al estilo de los metales (particularmente los objetos de oro), sugieren una ubicación tentativa entre los AD 800 y 1000. Dos fechados obtenidos de la excavación de un recinto localizado en la terraza superior (Alto de La Isla) brindó una 


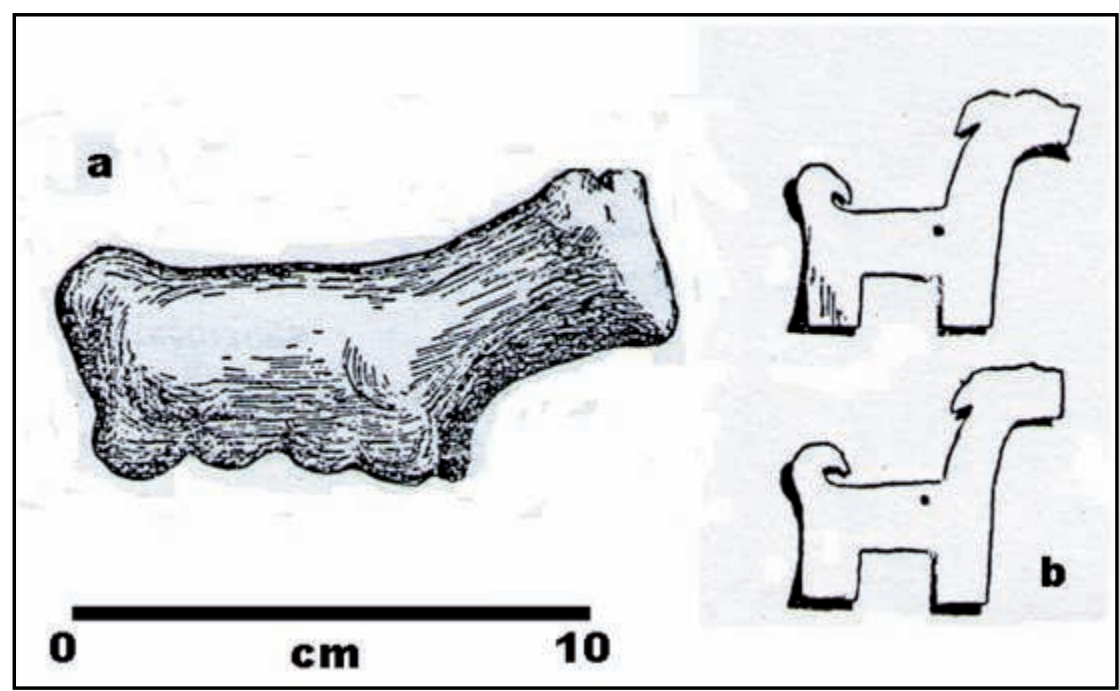

Figura 6. (a) Illa de terracota; (b) estatuillas de oro con forma de llamas. Dibujos originales de Debenedetti (1910).

cronología de entre AD 1029 y 1302 (Rivolta 2005). Sin embargo, más fechados son necesarios para poder determinar cronológicamente la variabilidad observada en los contextos de las inhumaciones.

La evidencia de San Pedro de Atacama y de otros sitios de la Quebrada de Humahuaca implica que este estilo cerámico estuvo involucrado en cierto tipo de tráfico. Datos provenientes de dos tumbas, Morro 11 (M11) y Necrópolis A 21 (Nea21), son sugerentes al respecto. Estas inhumaciones son diferentes al resto, en primer lugar, porque la cerámica no es la principal y más numerosa ofrenda y, por otra parte, fueron encontrados un amplio conjunto de diferentes tipos de artefactos, algunos de ellos con materias primas exóticas a la Quebrada y otros ligados al manejo de las llamas.

M11 tiene sólo un individuo inhumado con una gran variedad de artefactos, muchos de ellos exóticos, tales como mineral de cobre, artefactos metálicos, siluetas de llamas hechas de oro, largas hojas de oro (algunos de ellos quizás sean preformas de llamas) y campanas (ver análisis detallado en Tarragó et al. 2010). Se destaca un esqueleto de loro (Ara sp., c.f. chloroptera) de las tierras bajas. También hay objetos de hueso como una horqueta de atalaje o tarabita (ver Raviña et al. 2007) cuya función es atar la carga a las llamas y una espátula, cucharas y tubos de hueso relacionados con el complejo alucinógeno y un cuerno de cérvido. También fue recuperada una estatuilla confeccionada en terracota conocida como illa. Las illas son objetos ceremoniales ligados a ritos de pastores para promover la reproducción y supervivencia del rebaño (Mariscotti de Görlitz 1978: 172) (Figura 6).

El interesante y detallado artículo de Tarragó et al. (2010) sobre la tumba contiene una serie de interpretaciones del contexto que debemos atender. Refiriéndose a los objetos de oro ${ }^{1}$ expresan que "...sólo pudieron pertenecer a sujetos de rango que tenían acceso a materiales privilegiados y al tráfico de larga distancia" (p. 59) en un ámbito de creciente diferenciación social, cuestión que parece avalada por la evidencia. Estiman, además, que el individuo inhumado "...estuvo en vida relacionado con actividades metalúrgicas" (p. 61) en base al mineral de cobre presente, la "posible" escoria de fundición y otros elementos 
como el cuerno de cérvido que pudo, según los autores, estar relacionado con esta clase de actividades. Sin embargo, pensamos que el peso de la evidencia revisada, tales como las figurinas de llamas, la illa que es un artefacto específicamente pastoril, la tarabita y otros elementos que sugieren el manejo de llamas cargueras (campanillas), sumado a la cantidad de elementos exóticos, permiten plantear como alternativa, que el individuo inhumado fuera una temprana evidencia de una persona encargada del tráfico de bienes.

Otra tumba, Nea21, contiene evidencia que parece confluir en este sentido al igual que M11. Allí hubo tres individuos inhumados con un importante conjunto de elementos exóticos, tales como un nódulo de obsidiana, dos puntas de proyectil de esta materia prima, pigmento rojo, 8 placas de oro, un collar de malaquita y dos caracoles terrestres de las yungas, posiblemente Bulimus o Strophocheilus sp.

La asociación de estas dos tumbas y, posiblemente de M10 y Nea14 también, sugiere una temprana evidencia de individuos que estuvieron vinculados directamente al tráfico de bienes. Esta clase de evidencia ha sido registrada para momentos más tardíos en algunas localidades puneñas (Pérez de Micou 1997; Angiorama 2007). Entonces, si la evidencia de las tumbas M11 y Nea21 de La Isla han sido correctamente interpretadas, pueden mostrar la emergencia de individuos especializados en el tráfico de bienes, sosteniendo la opción 2.

\section{Conclusión}

Los casos aquí revisados muestran que durante tiempos formativos hubo un regular, aunque no muy intenso, sistema de intercambio. Este sistema tenía una gran área de distribución y una remarcable regularidad temporal como muestra la evidencia de la obsidiana. Más aún, hay ciertas cuestiones que permiten sostener que las caravanas de llamas fueron el principal vehículo responsable del intercambio, como había sido sugerido por Núñez y Dillehay (1995 [1978]) quienes hablaron a favor del funcionamiento de tempranos circuitos con ejes distantes activados a través de las caravanas. También Tarragó (1989) expresó que "tempranas poblaciones altas orientaban sus caravanas hacia ambientes con producciones exóticas orientales de desarrollo floreciente". Estos planteos han sido fuertemente sostenidos posteriormente con los resultados de los estudios sobre aprovisionamiento y distribución de las obsidianas (Yacobaccio et al. 2002).

Los ejemplos considerados en este trabajo permiten discutir algunas cuestiones relacionadas con diferentes escalas temporales. No podemos pensar en una estructura del tráfico circumpuneño de las mismas características durante 4000 años. Sin embargo, aunque no tenemos evidencia para efectuar un análisis detallado sobre este tema podemos plantear algunas hipótesis.

La distribución de las obsidianas es una imagen promedio para todo el período considerado y su esfera de interacción parece haber sido regular en el tiempo. Evidencias provenientes del denominado corredor Verde-Vilama, en el área altoandina, donde se encuentra la fuente de Zapaleri/Laguna Blanca, indica que éste es atravesado por rutas tempranas como lo indica una fecha del sitio Corrales de Huayllajara y la presencia de cerámicas Los Morros, Sequitor Gris Pulido e incisos (Nielsen 2006, 2011: 98). Parte de esta circulación de las obsidianas puede ser explicada por la modalidad de tráfico incorporado, pero los datos presentados por el mismo autor indican que la misma habría comenzado alrededor de $\mathrm{AD} 500$, lo que dejaría fuera a los momentos anteriores, ya que la obsidiana de la fuente 
mencionada está registrada en otras áreas desde, al menos, 2000 años AP. La circulación de esta materia prima y sus productos respondió, evidentemente, a diversas modalidades que coexistieron y cuyo predominio fue variable en el tiempo.

El caso de la cerámica San Francisco abarca el momento comprendido entre los 2040 y 1350 AP. El examen de la distribución de la misma es complejo, ya que incluye posiblemente difusión démica (Volcán) e intercambio. En este caso la curva fall-off señala que su distribución pudo obedecer al funcionamiento de intercambios sucesivos a partir de centros secundarios de redistribución. Si esto es correcto los grupos puneños han sido los que activaron este tráfico promoviendo la amplia distribución de algunas modalidades decorativas de este estilo cerámico. La escasa representación de elementos alóctonos en el área de San Francisco brinda cierto apoyo a esta idea que, a su vez, se enmarca en la función redistributiva de los asentamientos-eje dentro del modelo de movilidad giratoria.

Hacia finales del período (AD 800-1000) el caso de La Isla señala el hecho de que la opción 2 (individuos especializados dentro de las comunidades pastoriles) es la más probable explicación sobre la naturaleza de los agentes involucrados en el tráfico de la misma, anunciando lo que sucederá en momentos más tardíos donde hubo un notable incremento en la intensidad del tráfico de bienes. La opción 2 apela al carácter social del sistema de la circulación de bienes sostenido por la teoría y por los resultados de las más recientes investigaciones en el tema (Núñez A. y Nielsen 2011) y que el desarrollo del mismo no estuvo acoplado a la expansión de la agricultura, sino del pastoreo de camélidos, como parece ilustrar específicamente el contexto de la tumba M11.

La evidencia muestra los complejos mecanismos involucrados en el tráfico de bienes y el estatus de los individuos (y grupos) responsables del mismo. Asimismo, se aprecia que el período no es un todo homogéneo impidiendo cualquier generalización que lo abarque en su totalidad (si es que podemos hablar de esto). Más bien, se observa que estuvo atravesado por momentos de cambio, pero todavía no tenemos una exacta dimensión acerca de su actuación. Tanto las fluctuaciones ambientales como los cambios sociales debieron tener una gran influencia sobre las rutas de tránsito y, más aún, la naturaleza misma del intercambio. En este punto se abre una ventana a partir de la cual asomarnos con mayor nivel de detalle a este apasionante tema.

\section{Agradecimientos}

Agradezco a Patricia Solá la confección de las Figuras 3, 4, 6 y 7. A Bibiana Vilá la foto de la Figura 1 y a los dos revisores anónimos que con sus comentarios contribuyeron a mejorar sustancialmente el trabajo. Como se dice habitualmente, todo error es mío.

\section{Notas}

${ }^{1}$ Los autores interpretan la actitud de las figurinas de llamas como representación probable "de un animal en celo" (p. 53) debido a la posición de la cola levantada y las orejas acostadas. Sin embargo, la posición que adopta la cabeza en un camélido en celo es con el hocico hacia arriba y el cuello estirado ("flehmen"). La posición de la cola para arriba y las orejas hacia atrás denota un comportamiento agresivo de tipo 1, que se da entre las hembras cuando pretenden generar espacio frente a otras hembras para poder alimentarse. Generalmente este comportamiento va acompañado de escupidas (Vilá 1990). 


\section{Bibliografía citada}

Angiorama, C.I.

2007. ¿Una ofrenda "caravanera" en Los Amarillos? Minerales y tráfico de bienes en tiempos prehispánicos. En: Producción y circulación prehispánicas de bienes en el sur andino (ed. por A.E. Nielsen, M.C. Rivolta, V. Seldes, M.M. Vázquez and P. Mercolli), pp. 383-391. Editorial Brujas, Córdoba.

Aschero, C.A.

2006. De cazadores y pastores. El arte rupestre de la modalidad río Punilla en Antofagasta de la Sierra y la cuestión de la complejidad en la Puna meridional argentina. En: Tramas en la Piedra. Producción y usos del arte rupestre (ed. por D. Fiore and M.M. Podestá), pp. 103140. AINA-World Archaological Congress-Sociedad Argentina de Antropologia, Buenos Aires.

Babot, M.P.

2004. Tecnología y Utilización de Artefactos de Molienda en el Noroeste Prehispánico. Tesis de Doctorado, Universidad Nacional de Tucumán, Tucumán.

Berenguer Rodríguez, J.

2004. Caravanas, Interacción y Cambio en el Desierto de Atacama. Sirawi Ediciones-Museo Chileno de Arte Precolombino, Santiago.

Carrier, J.G.

2008. Exchange. En: Handbook of Material Culture (ed. por C. Tilley, W. Keane, S. Küchler, M. Rowlands y P. Spyer), pp. 373-383. Sage, Los Angeles.

Cartajena, I.; L. Núñez y M. Grosjean

2007. Camelid domestication in the western slope of the Puna de Atacama, Northern Chile. Anthropologica 42: 155-174.

Cipolletti, M.S.

1984 Llamas y mulas, trueque y venta: el testimonio de un arriero puneño. Revista Andina 2: 513-538.

Cremonte, B. y M. Garay de Fumagalli

2001. Una Ocupación Temprana en el Pucará de Volcán (Dpto. Tumbaya, Jujuy). Actas del XIII Congreso Nacional de Arqueología Argentina, I: 157-171. Córdoba.

Cocilovo, J.A.; H.H. Varela y S.G. Valdano

2001. Estructura de la población antigua de la Quebrada de Humahuaca. En: Historia Prehispánica Argentina (ed. por E.E. Berberián and A. Nielsen), vol. I: 265-287. Editorial Brujas, Córdoba.

Debenedetti, S.

1910. Exploración Arqueológica en los Cementerios Prehistóricos de la Isla de Tilcara (Quebrada de Humahuaca, Provincia de Jujuy). Publicaciones de la Sección Antropológica 6, Facultad de Filosofía y Letras. Buenos Aires. 
Echenique, M. y J. Kulemeyer

2003. La excavación arqueológica de una "mancha blanca", el sector M43C en el sitio Moralito, Departamento de San Pedro, Provincia de Jujuy (República Argentina). En: La Mitad Verde del Mundo Andino (ed. por G. Ortiz and B. Ventura), pp. 99-129. Universidad Nacional de Jujuy, San Salvador.

Escola, P.

2004. Variabilidad en la explotación y distribución de obsidianas en la Puna Meridional argentina. Estudios Atacameños 28: 9-24.

Fernández Distel, A.A. 1998. Arqueología del Formativo en la Puna Jujeña. Colección Mankacén, Buenos Aires.

García, L.C.

2003. Azul Pampa en Etapas Productivas. Cuadernos FHyCS-UNJu, 20:15-35.

González, A.R. y J.A.Pérez

1972. Argentina Indígena. Vísperas de la Conquista. Paidós, Buenos Aires.

Lupo, L.; M.R. Morales; H.D. Yacobaccio; A. Maldonado y M. Grosjean 2007

Cambios ambientales en la Puna Jujeña durante los últimos 1200 años: explorando su impacto en la economía pastoril. XVI Congreso Nacional de Arqueología Argentina, Tomo III: 151-156.

Pacarina (número especial), Facultad de Humanidades y Ciencias Sociales, Universidad Nacional de Jujuy.

Malinowski, B.

1973[1922]. Los Argonautas del Pacífico Occidental. Ediciones Península, Barcelona.

Mauss, $\mathrm{M}$.

1971 [1923]. Sobre los dones y sobre la obligación de hacer regalos. En: Sociología y Antropología, pp. 155-263. Editorial Tecnos, Madrid.

Mariscotti de Görlitz, A.M.

1978. Pachamama Santa Tierra. Contribución al estudio de la religión autócotona en los Andes centro-meridionales. Indiana 8, Gebr. Mann Verlag, Berlin.

Muscio, H.J.

2007. Sociedad y mutualismo durante las expansiones agrícolas en entornos fluctuantes: un modelo de teoría evolutiva de juegos aplicado al doblamiento del período temprano de la Puna de Salta, Argentina. En: Producción y circulación prehispánicas de bienes en el sur andino (ed. por A.E. Nielsen, M.C. Rivolta, V. Seldes, M.M. Vázquez and P. Mercolli), pp. 105-134. Editorial Brujas, Córdoba.

Nielsen, A.E.

1997. El tráfico caravanero visto desde La Jara. Estudios Atacameños 14: 339-371.

2004. Aproximación a la Arqueología de la Frontera Tripartita Bolivia-Chile-Argentina. Chungara 36: 861-878. 
2006. Estudios internodales e interacción interregional en los Andes Circumpuneños: Teoría, método y ejemplos de aplicación. En: Esferas de Interacción Prehistóricas y Fronteras Nacionales Modernas: Los Andes Sur Centrales (ed. por H. Lechtman), pp. 29-62. IEP, Lima. 2011 El tráfico de caravanas entre Lípez y Atacama visto desde la Cordillera Occidental. En: En ruta: Arqueología, Historia y Etnografía del Tráfico Sur Andino (ed. por L. Núñez A, y A.E. Nielsen), pp. 83-110. Encuentro Grupo Editor, Córdoba.

Núñez, L.

1976. Geoglifos y tráfico de caravanas en el desierto chileno. Separata del tomo de homenaje al R.P. Gustavo Le Paige, pp. 147-201. Universidad del Norte, Chile.

Núñez, L. y T. Dillehay 1995 [1978]. Movilidad giratoria, armonía social y desarrollo en los Andes Meridionales: Patrones de tráfico e interacción económica. Universidad Católica del Norte, Antofagasta.

Núñez, L.; I. Cartajena; C. Carrasco y P. de Souza

2005. El templete de Tulán y sus relaciones formativas panandinas (norte de Chile). Bulletin de l'Institut Français de Études Andines 34: 299-320.

Núñez, L.; P. de Souza; I. Cartajena y C. Carrasco

2007. Quebrada Tulán: Evidencias de interacción circumpuneña durante el Formativo Temprano en el sureste de la cuenca de Atacama. En: Producción y circulación prehispánicas de bienes en el sur andino (ed. por A.E. Nielsen, M.C. Rivolta, V. Seldes, M.M. Vázquez and P. Mercolli), pp. 287-304. Editorial Brujas, Córdoba.

Núñez A., L. y A. E. Nielsen, editores 2011. En ruta: Arqueología, Historia y Etnografía del Tráfico Sur Andino. Encuentro Grupo Editor, Córdoba.

Núñez Regueiro, V.

1974. Conceptos Instrumentales y Marco Teórico en Relación al Análisis del Desarrollo Cultural del Noroeste Argentino. Revista del Instituto de Antropología V: 169-190. Córdoba.

Olivera, D.E.

2001. Sociedades agropastoriles tempranas: el formativo inferior del noroeste Argentino (ed. por E. Berberian y A. Nielsen), Historia Argentina Prehispánica, Vol. 1: 83-125. Editorial Brujas, Córdoba.

Olivera, D.E. y J.L. Grant

2009. Economía y Ambiente durante el Holoceno Tardío (ca- 4500-400) de Antofagasta de la Sierra (Puna Meridional Argentina). En: Temas de Arqueología 2: Estudios Tafonómicos y Zooarqueológicos (ed. por A. Acosta, D. Loponte y L. Mucciolo), pp. 97-129. INAPl, Buenos Aires.

Ortiz, G.

2003. Estado actual del conocimiento del denominado complejo o tradición cultural San Francisco, a 100 años de su descubrimiento. En: La Mitad Verde del Mundo Andino (ed. por G. Ortiz and B. Ventura), pp. 23-71. Universidad Nacional de Jujuy, San Salvador de Jujuy. 
2007. El paisaje macroregional. El uso del espacio social expandido a través de la circulación de objetos. En: Producción y circulación prehispánicas de bienes en el sur andino (ed. por A.E. Nielsen, M.C. Rivolta, V. Seldes, M.M. Vázquez y P. Mercolli), pp. 305-328. Editorial Brujas, Córdoba.

Paz Flores, M.P.

2000. Los llameros de Qochauma y sus viajes a Markapata. En: Pastoreo Altoandino: realidad, sacralidad, posibilidades (ed. por J.A. Flores Ochoa y Y. Kobayashi), pp. 135-148. Plural editores/MUSEF, La Paz.

Pimentel G., G. C. Rees, P. de Souza y L. Arancibia

2011. Viajeros Costeros y Caravaneros. Dos estrategias de movilidad en el Período Formativo del desierto de Atacama, Chile. En: En ruta: Arqueología, Historia y Etnografía del Tráfico Sur Andino (ed. por L. Núñez A, y A.E. Nielsen), pp. 43-82. Encuentro Grupo Editor, Córdoba.

Polanyi, K.

1977. The Livelihood of Man. Academic Press, New York.

Raffino, R. A.

1977. Las aldeas del Formativo Inferior de la quebrada del Toro (Salta, Argentina). Estudios Atacameños 5: 65-109.

Pérez de Micou, C.

1997. Indicios caravaneros en contextos funerarios de la Puna argentina. Estudios Atacameños 14: 143-158.

Raviña, M.G.; A.M. Fernández y A. Caparelli

2007. La relación de las tarabitas, horquetas o ganchos de atalaje con el tráfico de bienes en momentos tardíos prehispánicos. Estudios Atacameños: Arqueología y Antropología Surandinas 33: 87-104.

Rivolta, M.C.

2005. Cambio Social en la Quebrada de Humahuaca (1100-1400 d.C.). Instituto Interdisciplinario Tilcara, Facultad de Filosofía y Letras, Universidad de Buenos Aires.

Rydén, S.

1944. Contribution to the Archaeology of the Rio Loa Region. Elanders Bocktryckeri Aktiebolag, Göteborg.

Scattolin, M.C.

2007. De las comunidades aldeanas a los curacazgos en el Noroeste Argentino. Boletín de Arqueología PUCP 10: 357-398.

Seldes, V. y G. Ortiz

2007. Primeros resultados de estudios bioarqueológicos en poblaciones formativas de las yungas jujeñas. XVI Congreso Nacional de Arqueología Argentina, Tomo II: 327-332. Pacarina (número especial), Facultad de Humanidades y Ciencias Sociales, Universidad Nacional de Jujuy. 
Selenfreund, A.; M. Pino, M. D. Glascock, C. Sinclaire, P. Miranda, D. Pasten, S. Cancino, M. I. Dinator, y J. R. Morales

2010. Morphological and Geochemical Analysis of the Laguna Blanca/Zapaleri Obsidian in the Atacama Puna. Geoarchaeology: An International Journal 25: 245-263.

Smith, M.E. y P. Peregrine

2012. Approaches to Comparative Analysis in Archaeology. En: The Comparative Archaeology of Complex Societies (ed. por M.E. Smith), pp. 4-20. Cambridge University Press, Cambridge.

Tarragó, M.

1977. Relaciones prehispánicas entre San Pedro de Atacama (Norte de Chile) y Relaciones Aledañas: La Quebrada de Humahuaca. Estudios Atacameños 5: 50-63.

1989. Contribución al conocimiento arqueológico de las poblaciones de los oasis de San Pedro de Atacama en relación con los otros pueblos puneños, en especial, el sector septentrional del Valle Calchaquí. Tesis Doctoral, Facultad de Humanidades y Arte (UNR). Rosario.

Tarragó, M.N., L. R. González, G. Ávalos y M. Lamamí 2010. Oro De Los Señores. La Tumba 11 de La Isla de Tilcara (Jujuy, Noroeste Argentino). Boletín del Museo Chileno de Arte Precolombino 15: 47-63.

Trigger, B.G.

2006. Understanding Early Civilizations. Cambridge University Press, Cambridge.

Torrence, R.

1996. TRADE, Prehistoric. En: The Oxford Companion to Archaeology (ed. por B. Fagan), pp. 718-720. Oxford University Press, New York-Oxford.

Valenzuela, D.; C.M. Santoro y L. Briones

2011. Arte Rupestre, Tráfico e Interacción Social: Cuatro Modalidades en el Ámbito Exorreico de los Valles Occidentales, Norte de Chile (Períodos Intermedio Tardío y Tardío, ca. 1000-1535 D.C.). En: En ruta: Arqueología, Historia y Etnografía del Tráfico Sur Andino (ed. por L. Núñez A, y A.E. Nielsen), pp. 199-245. Encuentro Grupo Editor, Córdoba.

Vilá, B.L.

1990. Comportamiento de la vicuña durante la temporada reproductiva. Tesis de Doectorado. Facultad de Ciencias Exactas y Naturales (UBA). Buenos Aires.

Yacobaccio, H.D. 2004

Social Dimensions of Camelid Domestication in the Southern Andes. Anthropozoologica 39: 237-247.

Yacobaccio, H.D.; P.S. Escola; M. Lazzari, y F. Pereyra

2002. Long-Distance Obsidian Traffic in Northwestern Argentina. En: Geochemical Evidence for Long-Distance Exchange (ed. por M.D. Glascock), pp. 167-203. Bergin and Garvey, Westport-London. 
Yacobaccio, H.D., P. S. Escola, F. X. Pereyra, M. Lazzari, y M. Glascock 2004. Quest for Ancient Routes: Obisidian Sourcing Research in Northwestern Argentina. Journal of Archaeological Science 31: 191-204.

Yacobaccio, H.D.

2006. Social Complexity in South Andean Hunting-Gathering Society. Section 17: Préhistoire de l'Amérique. C17.1: Change in the Andes: Origins of Social Complexity, Pastoralism and Agriculture. Acts of the XIVth UISPP Congress, University of Liège, Belgium. BAR International Series 1524, pp. 1-5. Oxford. 